\title{
Epidemiología de COVID-19 en un hospital de tercer nivel
} mexicano

César A. Arce-Salinas, ${ }^{1}$ Daniel López-Herrera, ${ }^{2}$ Adriana Hernández-Alarcón, ${ }^{3}$ Eva M. Luna-Rivera, ${ }^{4}$ Arely A. Bejarano-Juvera, ${ }^{5}$ Aditi K. Bustamante-Flores, ${ }^{5}$ Naomi Aguilar-Martínez, ${ }^{5}$ Jessica G. Azcorra-López, ${ }^{5}$ Berenice Cabañas-Espinosa, ${ }^{5}$ Yamile N. Esquivel-Torruco ${ }^{5}$ y Jesús Reyna-Figueroa ${ }^{5 *}$

${ }^{1}$ Dirección; ${ }^{2}$ Medicina del Trabajo; ${ }^{3}$ Servicio de Consulta Externa; ${ }^{4}$ Departamento de Enseñanza e Investigación; ${ }^{5}$ Pediatría. Petróleos Mexicanos, Hospital Central Sur de Alta Especialidad, Ciudad de México, México

\section{Resumen}

Introducción: A consecuencia de COVID-19, numerosos hospitales sufrieron una reconversión para la atención de esta enfermedad. Objetivo: Analizar el comportamiento epidemiológico hospitalario de COVID-19 entre marzo y agosto de 2020. Métodos: En una serie de casos se analizó el comportamiento epidemiológico de COVID-19 en un hospital de tercer nivel, para lo cual se estimaron frecuencias simples de casos, porcentajes e incidencia por cada 100 egresos hospitalarios. Resultados: De 491 sujetos con prueba positiva para SARS-CoV-2, 156 (31.7\%) fueron hospitalizados por datos clínicos de enfermedad moderada a grave. La edad promedio fue de 59.1 años; 121 casos (75 \%) egresaron por mejoría y 32 (20.5 \%), por defunción. El promedio de edad de quienes fallecieron fue de 69.7 años y el grupo etario más afectado fue el de 60 a 80 años (45.4\%). La letalidad calculada fue de 20.5 por 100 egresos hospitalarios, mientras que la calculada tomando en cuenta los pacientes positivos (ambulatorios y hospitalizados) fue de 6.5. Conclusiones: El comportamiento epidemiológico de COVID-19 fue similar al descrito en otros estudios; sin embargo, la letalidad y la mortalidad están por encima de la media nacional. Está pendiente el análisis de estas y de los factores que las favorecieron en nuestra población.

PALABRAS CLAVE: COVID-19. Mortalidad. Letalidad. México. Hospitalización.

\section{COVID-19 epidemiology in a Mexican tertiary care hospital}

\section{Abstract}

Introduction: As a result of COVID-19, many hospitals underwent a conversion for the care for this disease. Objective: To analyze COVID-19 hospital epidemiological behavior from March to August 2020. Methods: Through a series of cases, COVID-19 epidemiological behavior at the hospital was analyzed, for which simple case rates, percentages and incidence of COVID-19 per 100 hospital discharges were estimated. Results: Out of 491 subjects who tested positive for SARS-CoV-2, $156(31.7 \%)$ were hospitalized for clinical data of moderate to severe disease. Average age was 59.1 years; 121 cases (75\%) were discharged due to improvement, and 32 (20.5\%), due to death. Average age of those who died was 69.7 years, and the most affected age group was 60 to 80 years (45.4\%). Calculated lethality was 20.5 per 100 hospital discharges, while that calculated taking into account positive patients (outpatients and hospitalized patients) was 6.5. Conclusions: COVID-19 epidemiological behavior was similar to that described in other studies; however, lethality and mortality are above national average. The analysis of this and of the factors that favored it in our population is pending.

KEY WORDS: COVID-19. Mortality. Lethality. Mexico. Hospitalización. 


\section{Introducción}

En diciembre del 2019 surgió en la ciudad de Wuhan, provincia de Hubei en China, un brote de neumonía de etiología desconocida que a la postre fue denominada COVID-19, al identificarse en enero de 2020 al nuevo coronavirus SARS-CoV-2 como su agente causal. ${ }^{1}$ Para marzo de 2020, COVID-19 fue declarada pandemia por la Organización Mundial de la Salud, lo que marcó el inicio de una serie de estrategias para su contención y mitigación; entre ellas, el distanciamiento social y el confinamiento en más de 170 países. ${ }^{2,3} \mathrm{~A}$ nivel hospitalario, un número importante de instituciones de todos los países sufrieron una reconversión para dar prioridad a la atención de pacientes infectados por SARS-COV-2: incrementaron el número de camas útiles, superaron la capacidad de sus terapias intensivas y adquirieron ventiladores, esperando tener la capacidad de respuesta $\sin$ que su operatividad se viera rebasada; ${ }^{4}$ además, fortalecieron sus sistemas de vigilancia y adoptaron diferentes estrategias de contención. ${ }^{5}$

En México, la vigilancia epidemiológica de COVID19 se ha realizado a través del trabajo organizado y diligente de los servicios de salud de todas las entidades federativas, con profesionales dedicados a obtener información completa de los pacientes a partir del estudio epidemiológico de caso sospechoso de enfermedad respiratoria viral, el cual aplica a toda persona que cumpla con la definición operacional vigente, con la posterior notificación a la plataforma informática del Sistema Nacional de Vigilancia Epidemiológica, gestionado por la Dirección General de Epidemiología de la Secretaría de Salud y que está sustentado en los artículos 3, 137, 138 y 142 de la Ley General de Salud ${ }^{6}$ y apartados 6.5, 6.5.1 y 6.5.2 de la Norma Oficial Mexicana NOM-017-SSA-2-2012 para la vigilancia epidemiológica. ${ }^{7}$ Este sistema es capaz de analizar los datos y proporcionar una visión sólida que permita iniciar, profundizar o rectificar acciones de prevención y control; además, establece qué enfermedades están sujetas a notificación e investigación obligatorias, entre ellas los padecimientos ocasionados por el virus de la influenza.

En tanto que la definición operativa de COVID-19 ha evolucionado de acuerdo con el comportamiento epidemiológico y los hallazgos científicos respecto a su trasmisión, geolocalización y cuadro clínico, los casos confirmados establecen la positividad de una prueba de biología molecular.
La vigilancia epidemiológica intrahospitalaria y el reporte a los sistemas de información es responsabilidad de un centro, integrado por expertos en infectología y epidemiología, así como por personal médico-administrativo; además, debe cubrir varios objetivos, entre ellos, mantener actualizada la información y hacer los cambios necesarios. ${ }^{8}$ El número de publicaciones que abordan algún tema específico acerca de la pandemia de COVID-19 se ha incrementado exponencialmente en los últimos meses. Aun así, son escasas las series de casos de pacientes hospitalizados en forma secuencial por COVID-19 que ha sido confirmada ${ }^{9}$ y en México son prácticamente nulas. Por ello, el objetivo del estudio fue analizar el comportamiento epidemiológico poblacional de COVID-19 en el Hospital Central Sur de Alta Especialidad de Petróleos Mexicanos, entre marzo y agosto de 2020.

\section{Métodos}

Para hacer un comparativo del comportamiento epidemiológico de COVID-19 en el Hospital Central Sur de Petróleos Mexicanos con los datos informados a nivel nacional y por los servicios de salud de Petróleos Mexicanos, se revisaron las siguientes fuentes:

- Reportes semanales de casos de COVID-19, publicados en el Boletín Epidemiológico de la Dirección General de Epidemiología de la Secretaría de Salud.

- Informes epidemiológicos diarios de los servicios de salud de Petróleos Mexicanos.

- Base de datos del Hospital Central Sur de Alta Especialidad de Petróleos Mexicanos.

La sospecha de COVID-19 se consideró de acuerdo con la definición operativa inicial de la Secretaría de Salud Federal, que consideraba caso sospechoso a la persona de cualquier edad que presentara enfermedad respiratoria aguda leve o grave y que contara con alguno de los siguientes antecedentes hasta 14 días antes del inicio de síntomas:

- Haber estado en contacto con un caso de COVID-19, confirmado o bajo investigación.

- Viaje o estancia a países con transmisión local comunitaria de COVID-19 (China, Hong Kong, Corea del Sur, Japón, Italia, Irán y Singapur).

$Y$ posteriormente se agrego el siguiente criterio:

- Presentar en los últimos siete días al menos dos de los siguientes signos y síntomas: tos, fiebre 0 cefalea (dolor de cabeza intenso y persistente) acompañados de al menos uno de los siguientes 
signos o síntomas: dificultad para respirar, falta de apetito, dolor de articulaciones o músculos, conjuntivitis, dolor de garganta y congestión nasal.

Como caso confirmado se definió al paciente con resultado positivo de la reacción en cadena de la polimerasa realizada en el laboratorio institucional, avalado por el Instituto de Diagnóstico y Referencia Epidemiológicos "Dr. Manuel Martínez Báez".

Para el análisis se estimaron frecuencias simples de casos, porcentajes e incidencias de COVID-19 por cada 100 egresos hospitalarios mensuales. Para estimar la tendencia de la incidencia se utilizó un coeficiente de correlación (no paramétrico) entre las semanas y la incidencia mensual. El cambio mensual se estimó mediante regresión lineal simple. Las incidencias del hospital se compararon con las incidencias institucionales y nacionales mediante intervalos de confianza de $95 \%$. Para el cálculo de la mortalidad bruta se obtuvo el cociente de defunciones por COVID-19 entre la población derechohabiente al Hospital Central Sur, el cual se multiplicó por 1000. La tasa de letalidad se calculó con la proporción de defunciones entre los casos positivos a SARS-CoV-2 por 100.

\section{Resultados}

De 491 sujetos con prueba positiva a SARS-CoV-2, $156(31.7 \%)$ fueron hospitalizados por datos clínicos de COVID-19 moderada a grave; $103(66 \%)$ correspondieron al sexo masculino y $53(34 \%)$, al sexo femenino. La edad promedio de los pacientes fue de $59.1 \pm 15.4$ año. El mayor número de casos se notificó en el grupo de 41 a 60 años, con $41 \%$; le siguieron los grupos de 61 a 80 años, con $39.1 \%$; de 21 a 40 años, con $9.6 \%$; de 81 o más años, con $8.9 \%$; y de 0 a 20 años, con $1.2 \%$ (un caso de siete años y otro de 14).

Ciento veintiún casos (75\%) fueron egresados por mejoría, $32(20.5 \%)$ por defunción, dos pacientes fueron trasladados a otra institución y uno se egresó voluntariamente. El tiempo de hospitalización promedio fue de $12 \pm 10.2$ días.

El promedio de edad de quienes fallecieron fue de $69.7 \pm 12.9$ años; el grupo de edad mayormente afectado fue el de 60 a 80 años, con $45.4 \%$ de los casos, seguido de los grupos de 40 a 60 años y de 80 o más, cada uno con $27.2 \%$.

De acuerdo con el sitio de detección y atención inicial, 147 de los casos (97\%) fueron identificados en la Clínica de Atención de Enfermedades Respiratorias; los restantes nueve fueron identificados en la Unidad de Terapia Intensiva $(n=2)$, en los servicios de Medicina Interna $(n=3)$, Cirugía General $(n=1)$, Urgencias $(n=2)$ y Nefrología $(n=1)$.

\section{Hospitalizaciones}

El promedio de hospitalizaciones por mes fue de 33.6 casos en el periodo de abril a agosto de 2020. La menor frecuencia correspondió al mes de abril, con siete casos (4.4\%), y la mayor correspondió a mayo, con 52 casos (33.3\%). Las cifras notificadas de junio a agosto correspondieron a $57.7 \%$ de los casos, lo que equivalió a un aumento de $20.6 \%$ respecto al trimestre anterior $(p=0.05)$, como puede apreciarse en la Figura 1, en la que también puede observarse la tendencia creciente de pacientes hospitalizados por COVID-19: de $50 \%$ de abril a agosto ( ho de Spearman $=0.77, p=0.001$ ).

\section{Letalidad y mortalidad}

La letalidad calculada en los pacientes atendidos en el Hospital Central Sur de Alta Especialidad fue de 20.5 por 100 egresos hospitalarios, mientras que la calculada tomando en cuenta a los pacientes positivos (ambulatorios y hospitalizados) fue de 6.5 por cada 100 pacientes. La mortalidad fue notificada con una tasa de 1.1 por 1000 usuarios del sistema de salud de Petróleos Mexicanos. De los meses de estudio, agosto presentó el mayor porcentaje de defunciones, con $40 \%(n=15)$; seguido de junio, con $25.6 \%$, julio con $23.1 \%$ y abril con $14.3 \%$ (Figura 2).

\section{Discusión}

El Hospital Central Sur de Alta Especialidad es parte de los servicios de salud de Petróleos Mexicanos; se trata de un centro de tercer nivel de atención con una población estimada de 27000 derechohabientes. Para la atención de pacientes afectados por la pandemia de COVID-19, en abril de 2020 fue reconvertido conforme el acuerdo nacional de atención del sistema de salud mexicano.

Los datos de mayor interés encontrados en nuestra serie son las tasas de letalidad y de mortalidad bruta reportadas en el hospital. Comparativamente, la letalidad en pacientes hospitalizados fue $100 \%$ 


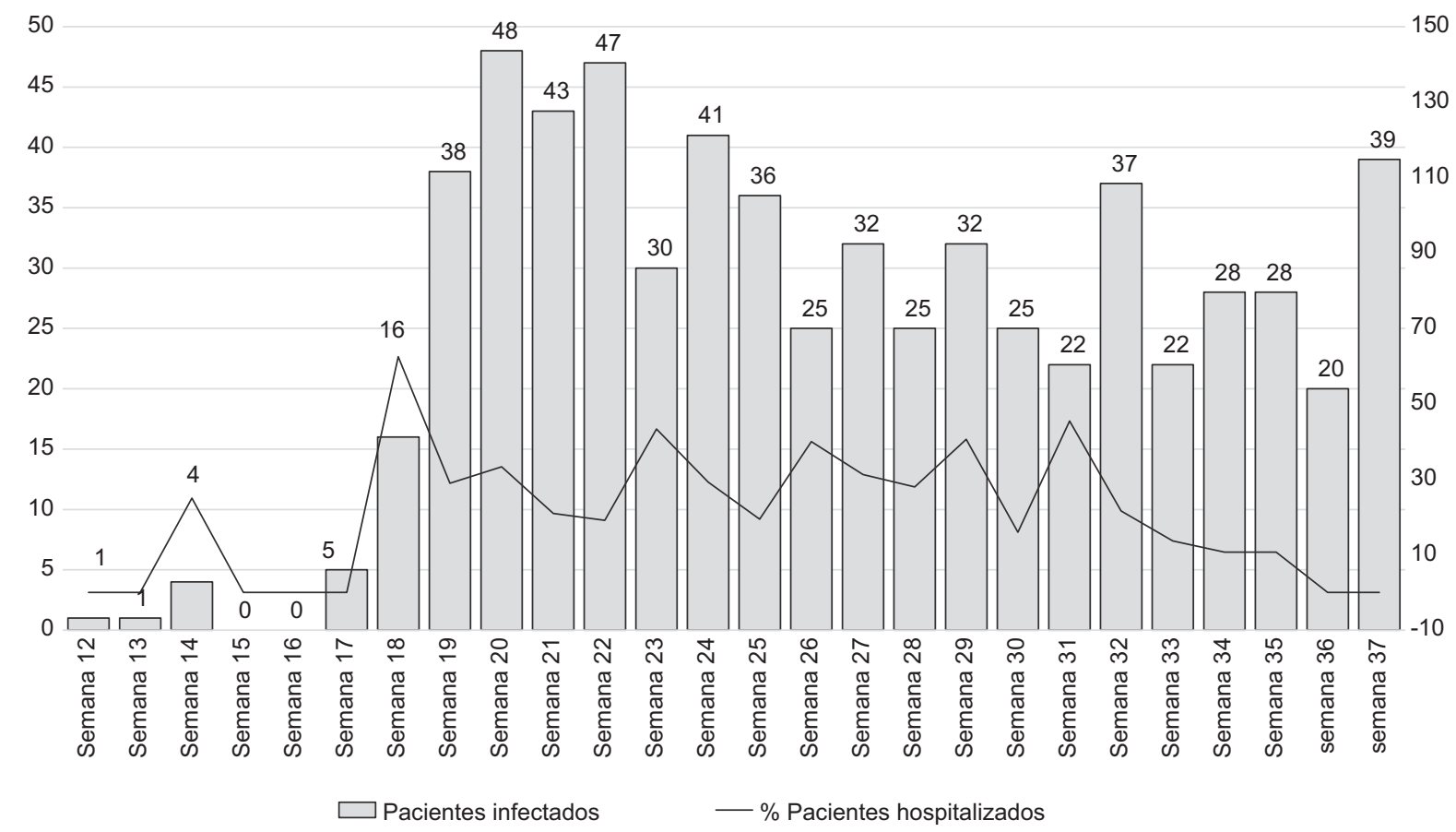

Figura 1. Porcentaje de pacientes hospitalizados por COVID-19 en relación con los pacientes con prueba positiva atendidos en el Hospital Central Sur de Alta Especialidad.

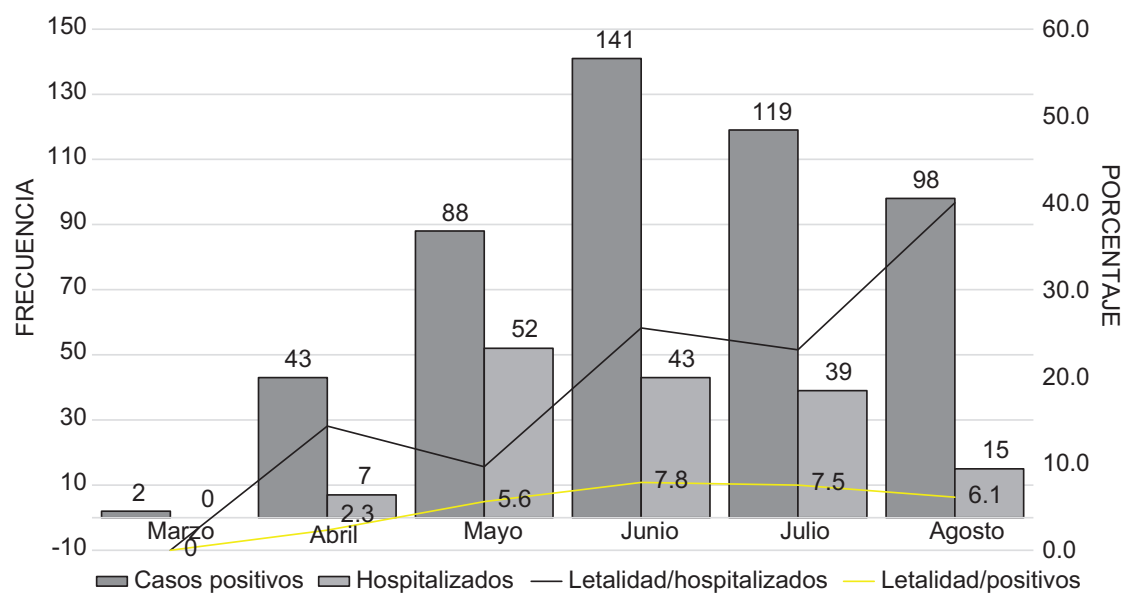

Figura 2. Tasa de letalidad por mes en el Hospital Central Sur de Alta Especialidad, conforme las muertes/casos positivos (incluidos los pacientes ambulatorios) y las muertes/hospitalizaciones.

mayor que la reportada en el país y $20 \%$ mayor que la registrada por los servicios de salud de Pemex. Sin embargo, al analizar la letalidad de los pacientes con COVID-19 y tomando en cuenta los pacientes positivos hospitalizados y atendidos ambulatoriamente, las cifras cambian diametralmente: resultó ser $61.9 \%$ menor respecto a la tasa nacional y $39.6 \%$ menor a las reportadas en el resto de los servicios de salud de Petróleos Mexicanos. La mortalidad fue mayor que la reportado en ambos casos y la comparación gráfica puede observarse en la Figura 3. Algunas explicaciones pueden darse desde el punto de vista poblacional y sus características. Al respecto, algunos autores mencionan que la letalidad de COVID-19 depende de la gravedad del caso; particularmente el Hospital Central Sur de Alta 


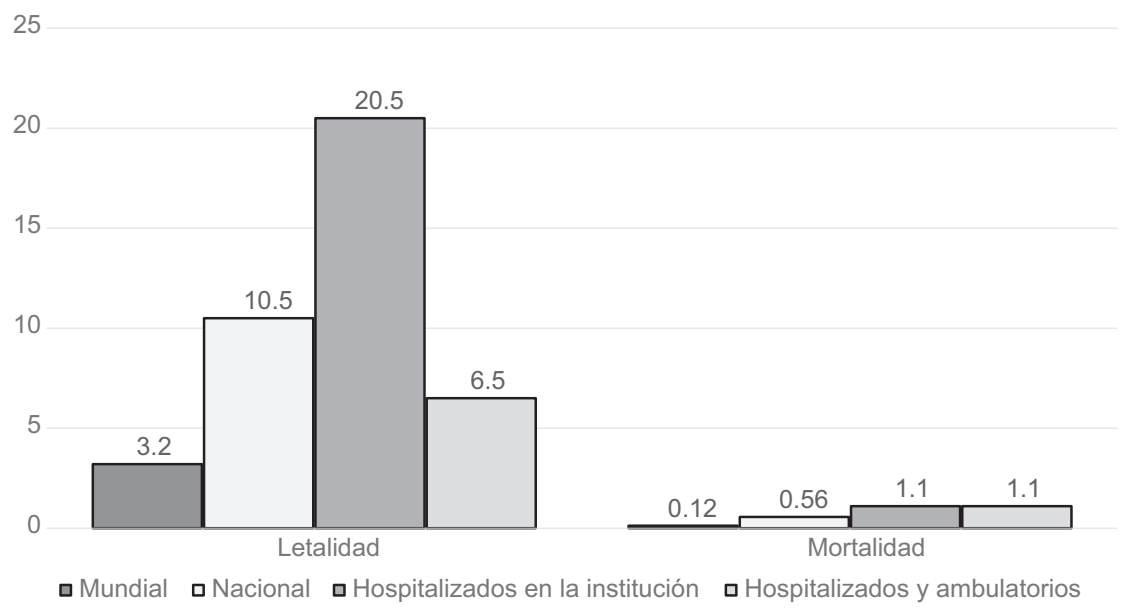

Figura 3. Comparativo de la mortalidad y letalidad del Hospital Central Sur con tasas mundiales, nacionales y de los servicios de salud de Petróleos Mexicanos.

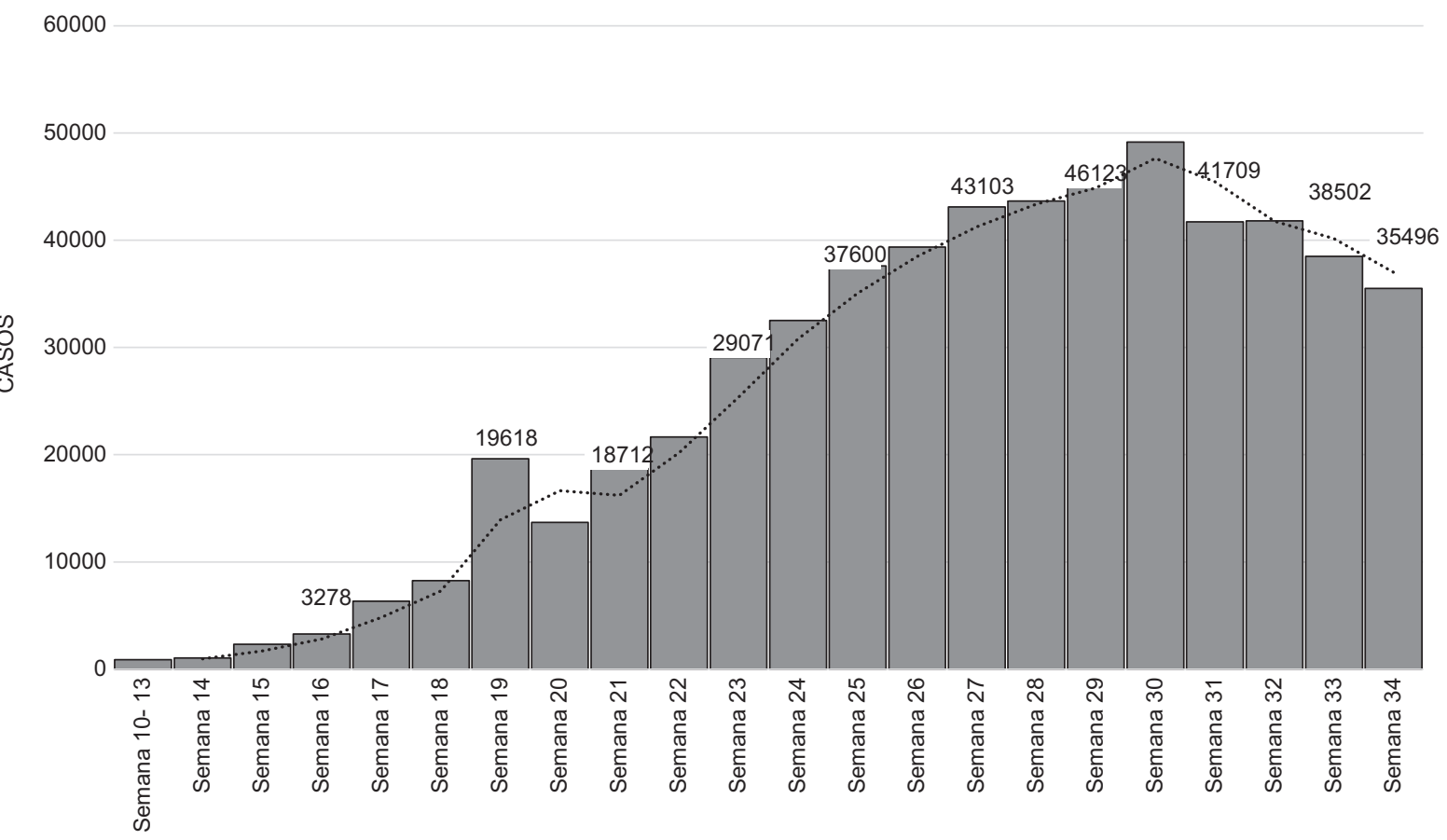

Figura 4. Número de casos en México de la semana epidemiológica 10 a la 34 (de marzo a agosto de 2020). Fuente: Boletín Epidemiológico Sistema Nacional de Vigilancia Epidemiológica Sistema Único de Información, Dirección General de Epidemiología.

Especialidad admitió para hospitalización a pacientes con cuadros graves y muy graves, estrategia adoptada (junto con el seguimiento diario de pacientes positivos confinados en su hogar para detectar complicaciones) para evitar la saturación hospitalaria. La tasa de letalidad en nuestra serie fue similar a la de hospitales que atienden pacientes con enfermedades graves asociadas, como el cáncer, en quienes se ha descrito hasta $28.6 \% .^{10}$ Sin embargo, el diseño de esta serie no puede abordar la complejidad de esta situación y los resultados no pretenden hacer un análisis de las causas. 
Para septiembre de 2020, los datos epidemiológicos mundiales indicaron que COVID-19 había sido responsable de casi un millón de muertes (3.2\%) de un total de treinta y un millones de casos. En México, de acuerdo con las notificaciones analizadas, hasta el 22 de septiembre se reportaron 684113 casos y 72179 muertes, con una tasa de letalidad de 10.5 por 100 casos,$^{11}$ y una mortalidad bruta de 0.56 por 1000 habitantes (cálculo en el que se consideraron ciento veintisiete millones de habitantes en México de acuerdo con el último reporte del Instituto de Nacional de Estadística y Geografía). Hasta la semana epidemiológica 35 de 2020 (correspondiente a la última semana de agosto) se reportaron 573885 casos confirmados; en la semana 29 ocurrió el mayor número de casos del periodo analizado (Figura 4).

En los servicios de salud de Petróleos Mexicanos, de acuerdo con cifras oficiales, hasta el 30 de agosto de 2020 se notificaron 7661 casos de COVID-19 posterior a la realización de 12393 pruebas (positividad de $61.8 \%$ ), 1258 defunciones (letalidad de 16.4 por 100 casos positivos) y una tasa bruta de mortalidad de 1.6 personas por 1000 (calculo que tomó en cuenta 765000 derechohabientes).

La media de edad fue similar a la de otras series si se considera el análisis intercuartílico, ${ }^{9,12}$ en tanto que el porcentaje de hospitalizaciones fue similar al reportado en otros análisis. ${ }^{13}$

\section{Limitaciones}

Este estudio tiene varias limitaciones. En primer lugar, la población del estudio solo incluyó pacientes del área de influencia del Hospital Central Sur de Alta Especialidad; en segundo lugar, los datos se recopilaron de la base de datos electrónica de registros sanitarios, lo que impidió el nivel de detalle posible con una revisión manual de registros médicos. En tercer lugar, las estadísticas descriptivas de los subgrupos no se ajustaron a los posibles factores de confusión ni se pretendió describir a la población ni sus características demográficas.

\section{Conflicto de intereses}

Los autores declaran no tener conflicto de intereses alguno.

\section{Financiamiento}

La presente investigación no recibió ninguna beca específica de agencias de los sectores públicos, comercial, o sin ánimo de lucro.

\section{Responsabilidades éticas}

Protección de personas y animales. Los autores declaran que para esta investigación no se realizaron experimentos en seres humanos ni en animales.

Confidencialidad de los datos. Los autores declaran que en este artículo no aparecen datos de pacientes.

Derecho a la privacidad y consentimiento informado. Los autores declaran que en este artículo no aparecen datos de pacientes.

\section{Bibliografía}

1. Kannan S, Shaik Syed Ali P, Sheeza A, Hemalatha K. COVID-19 (novel coronavirus 2019) - recent trends. Eur Rev Med Pharmacol Sci. 2020;24:2006-2011.

2. Sohrabi C, Alsafi Z, O'Neill N, Khan M, Kerwan A, Al-Jabir A, et al. World Health Organization declares global emergency: A review of the 2019 novel coronavirus (COVID-19). Int J Surg. 2020;76:71-76.

3. Cruz AT, Zeichner SL, COVID-19 in children: initial characterization of the pediatric disease. Pediatrics. 2020;145:e20200834.

4. Kashi AH. COVID-19, urologists and hospitals. Urol J. 2020;17:327.

5. Chang YT, Lin CY, Tsai MJ, Hung CT, Hsu CW, Lu PL, Hou MF. Infection control measures of a Taiwanese hospital to confront the COVID-19 pandemic. Kaohsiung J Med Sci. 2020;36:296-304.

6. Ley General de Salud. México: Secretaría de Salud; 2020.

7. Norma Oficial Mexicana NOM-017-SSA2-2012, para la vigilancia epidemiológica. México: Diario Oficial de la Federación; 2013 Feb 19.

8. Lineamientos de reconversión hospitalaria. Versión 5 de abril, 2020. México: Secretaría de Salud; 2020.

9. Richardson S, Hirsch JS, Narasimhan M, Crawford JM, McGinn T, Davidson $\mathrm{KW}$, et al. Presenting characteristics, comorbidities, and outcomes among 5700 patients hospitalized with COVID-19 in the New York City Area. JAMA. 2020;323:2052-2059.

10. Zhang L, Zhu F, Xie L, Wang C, Wang J, Chen R, Jia $P$, et al. Clinical characteristics of COVID-19-infected cancer patients: a retrospective case study in three hospitals within Wuhan, China. Ann Oncol. 2020;31:894-901.

11. Coronavirus Resource Center, Universidad Johns Hopkins [Internet] Baltimore, EE. UU. Critical trends: tracking critical data. [Actualización 2020 Sep. 22]. Disponible en: https://coronavirus.jhu.edu/data

12. Superman G, Fidel RA, Mallete KM, Hammen $C$, Abdulá $H$, Ente $A$, et al. Clinical characteristics and morbidity associated with coronavirus disease 2019 in a series of patients in Metropolitan Detroit. JAMA Netw Open. 2020;3:e2012270.

13. Price-Haywood EG, Burton J, Fort D, Seoane L. Hospitalization and mortality among black patients and white patients with Covid-19. N Engl J Med. 2020;382:2534-2543. 